\title{
Review
}

\section{Clinical studies on rising and re-rising neurological diseases in Japan - A personal contribution -}

By Akihiro IGATA*1,†

(Communicated by Masanori OTSUKA, M.J.A.)

\begin{abstract}
Throughout my research life, I experienced to discover the causes of some neurological diseases in Japan.

1) SMON (subacute myelo-optico-neuropathy). Since the early $1960 \mathrm{~s}$, a peculiar neurological disease became prevalent throughout Japan. Through the chemical analysis of the green urine, characteristic of this disease, it was found that this disease was caused by intoxication of the administered clioquinol, an anti-diarrheal drug. This discovery is a big topic in the history of Japanese medicine.

2) In early 1970s, I experienced many young patients with oedema and polyneuropathy in Kagoshima. Finally it was found that the disease was the long-forgotten beriberi, which had disappeared several decades ago. We must always be aware of beriberi even now, as far as we eat well-polished rice.

3) In 1972, we noticed a group of sporadic paraparesis in Kagoshima, which was 20 years later confirmed to be induced by human T lymphotropic virus type-I (HTLV-I). We named this disease as "HTLV-I associated myelopathy" (HAM). It gave a strong impact that the causative virus of adult T cell leukemia (ATL) can induce entirely different diseases, in terms of both the clinical course and the pathological features. It was also proven that HAM was identical with tropical spastic paraparesis, (TSP), which had been prevalent in many areas of tropical zones.

These experiences are good examples of our slogan "to keep in mind to send message of scientific progress from the local area to the international stage".
\end{abstract}

Keywords: SMON(subacute myelo-optico-neuropathy), beriberi, HAM (HTLV-I associated myelopathy)

Throughout my research life, I experienced some nice chances to discover the causes of neurological diseases in Japan. Here, some topics will be presented.

\section{1. $\mathrm{SMON}$}

(subacute myelo-optico-neuropathy) ${ }^{1)-3)}$

1.1. Introduction. Since early $1960 \mathrm{~s}$, a peculiar neurological disease became prevalent in many

*1 Nagoya University of Arts and Sciences, Aichi, Japan.

$\dagger$ Correspondence should be addressed: A. Igata, Nagoya University of Arts and Sciences, 57 Takenoyama, Iwasaki-cho, Nisshin, Aichi 470-0196, Japan.

Abbreviations: SMON: subacute myelo-optico-neuropathy; TPP: Thimaine pyro-phosphate; HAM: HTLV-1 associated myelopathy; HTLV-I: Human T lymphotropic virus type 1; ATL: Adult T cell Leukemia; ATLL: Adult T cell Leukemia/Lymphoma; TSP: Tropical spastic paraparesis. areas, and later rather pandemic throughout Japan. In 1963-64, I experienced an outbreak of this disease, mainly in the summer season, in Toda-city adjacent to the northern border of Tokyo, the number of which was more than 50 patients. ${ }^{1)}$ A few years thereafter, the number of the patients throughout Japan had increased to more than 10,000 by the end of 1970.2$)$

1.2. Clinical signs and symptoms of SMON. ${ }^{1), 2)}$ The clinical features are summarized as follows:

1) Onset was rather subacute, sometimes with relapses.

2) Abdominal symptoms (abdominal pain, diarrhea etc.) usually preceded the neurological ones.

3) Main neurological symptoms were symmetrical 


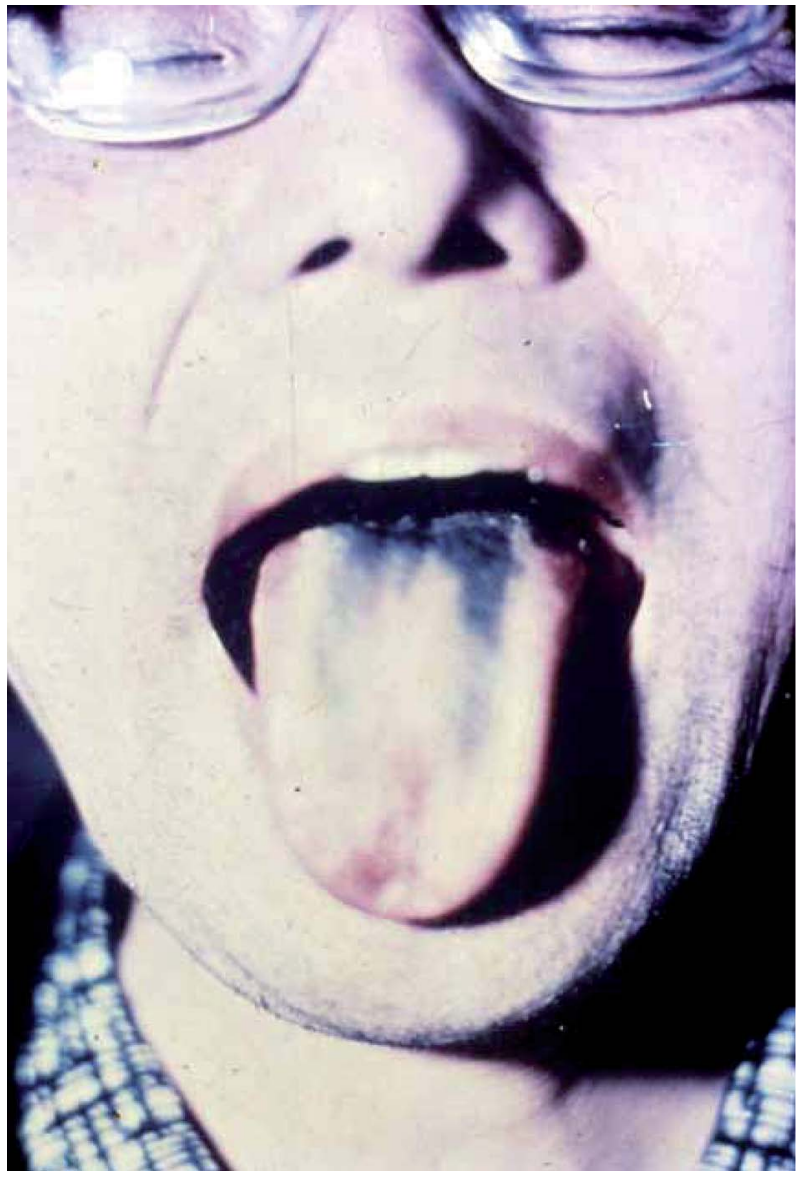

Fig. 1. The green tongue-fur of a patient with SMON. The green tongue-fur was observed frequently among SMONpatients. ${ }^{1)}$

paresthesia and hypoesthesia, predominant in the distal parts of the lower extremities, without any clear upper margin. In severe cases, the upper extremities were also involved.

4) Slight motor disturbance of the lower extremities.

5) Bladder and colon disturbances in severe cases.

6) Pyramidal signs with diminished Achilles tendon reflexes in many cases.

7) Visual disturbance in $20 \%$ of cases.

8) No signs of infection, no abnormal findings in blood and cerebrospinal fluid (CSF) suggesting the infection.

9) Rare in children, and both males and females were affected.

10) Death occurred sometimes with cerebral symptoms.

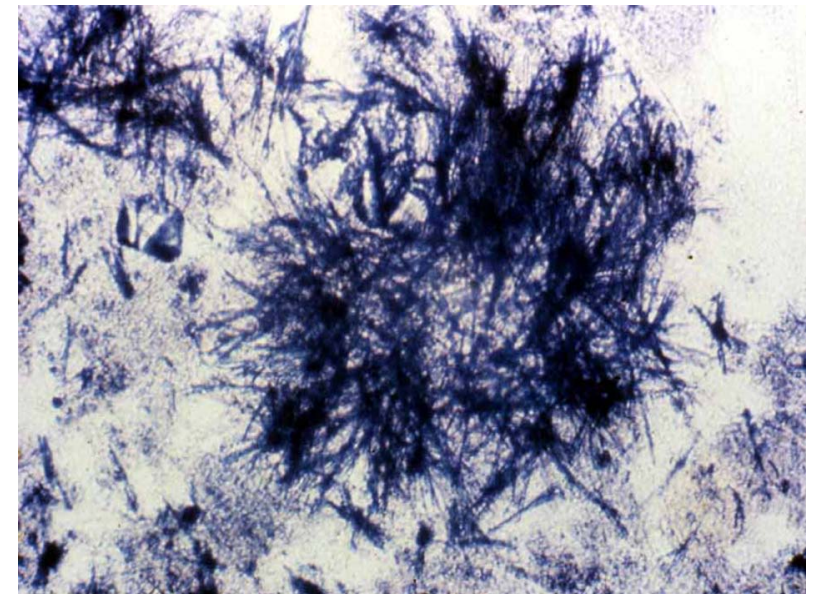

Fig. 2. Clioquinol conjugation with ferric ion in green urine. ${ }^{1), 6}$ )

At the beginning, this new disease was named "non-specific encephalo-myelitis" by the research group (Director Prof. M. Maekawa of Kyoto University), but due to the complexity, the name of SMON (subacute myelo-optico-neuropathy) became widely accepted, which was proposed by Prof. Yasuo Toyokura, Department of Neurology, University of Tokyo. ${ }^{4)}$ The name of SMON was based on its mode of onset and on the pathological findings, which were characterized by the pseudo-systemic degeneration of long tracts of the spinal cord, along with the involvement of the visual and peripheral nervous systems.

1.3. The endeavor to elucidate the cause of SMON. We were engaged in the research on the causes and management toward a new disease, but they remained obscure for several years. In 1970, Dr. Yukishige Inoue of Kyoto University ${ }^{5}$ suggested that a virus might be the responsible pathogen, inducing SMON but without any supportive evidence. It was a surprising topic, so that many patients with SMON became despaired to commit suicide.

Opposed to the virus hypothesis, we were convinced that it was not an infectious disease, from its clinical and pathological findings. In the same year, we found high incidence of the characteristic green tongue-fur (Fig. 1) among patients of SMON. Green feces were also found to be characteristic. ${ }^{1)}$

By chance in 1970, the green urine, excreted by 2 patients with $\mathrm{SMON}^{1), 6}$ ) was found. From these findings, we suspected that the green pigment might be the causative substance of SMON.

Through its chemical analysis by Prof. Zenzo Tamura and Dr. Sadanori Yoshioka, ${ }^{7)}$ the green pig- 
ment in urine was confirmed to be the clioquinol (5chloro-7-iodo-8-quinolinol, chinoform) excreted in combination with coexisting ferric-ion (Fig. 2). Clioquinol is a most popular anti-diarrheal agent (Enterovioform, Mexaform etc.). In these cases, iron-tablets were coincidently given for the treatment of anemia. It was surprising that clioquinol was found in the urine because it had been clearly written in all medical textbooks at that time, that it was insoluble in water and therefore not absorbed in intestine. These descriptions were proven later to be practically wrong, because more than $70 \%$ of orally administered clioquinol was found to be absorbed. ${ }^{2}$ )

The green urine was an exception because excreted clioquinol appeared colorless which turned green under the condition of co-existing iron-ion. Through this coincidental and serendipitious discovery, clioquinol was first nominated as a possible causative agent of SMON.

Following this discovery, I confirmed in a private hospital that the SMON occurred in $43.6 \%$ among 78 patients, to whom clioquinol was administered, whereas no SMON occurred among 77 patients without clioquinol administration. ${ }^{1)}$

Prof. Tadao Tsubaki, Department of Neurology, Niigata University, also became independently confident that clioquinol should be the causative agent of SMON and he directly recommended the Ministry of Health and Welfare of the Japanese Government not to use clioquinol throughout Japan. ${ }^{8)}$ Needless to say, I agreed with his proposal, which was later accepted by the Central Drug Affairs Council of the Japanese Government.

Hereafter the epidemiological studies throughout Japan were undertaken by a nation-wide research group but the hot discussions concerning its cause continued.

In this situation, I continued to report much data successively to support the clioquinol intoxication hypothesis. ${ }^{1)}$

As to why clioquinol can be the causative agent of SMON, whereas clioquinol was administered for the abdominal symptoms of it, it was clarified that these abdominal symptoms consisted of 2 components, the former was the non-specific abdominal symptoms, which initiated clioquinol administration and the latter was ones induced by the toxic effect of clioquinol as autonomic dysfunctions. ${ }^{1)}$

Concerning the rarity of SMON in children, I found that the duration of clioquinol administration in children was commonly very short, usually within
3 days. This was the main reason, why it was rare in children. ${ }^{1)}$

On the contrary, clioquinol administration to adult diarrheal patients was commonly for 4 weeks. It was the reason why it occurred mainly in adults. In addition, the higher dosage of more than $0.5 \mathrm{~g} / \mathrm{d}$ was also common, under the advertisement that "more dosage will be more effective" by pharmaceutical companies. 1)

As to the dose-response relationship, we confirmed from the clinical data that the low dosage and the short duration of administration (lower than $0.5 \mathrm{~g} / \mathrm{d}$, as a daily dosage and within 4 weeks) did not induce SMON. This finding clearly explained the reason why SMON occurred so rare in European countries where this clioquinol was widely used with strictly regulated doses. ${ }^{1), 9)}$

In addition we succeeded to experimentally induce the peripheral nerve lesions in rabbits by intravenous injection of clioquinol-emulsion, which were similar to those of SMON from every view points. ${ }^{1), 10)}$

1.4. The general agreement.2),3),10) In spite of our convincing data, the final agreement could not be obtained for a long time. In this situation Dr. Morizo Ishidate, the president of the Central Drug Affairs Council of the Japanese Governmental decided to stop the marketting of clioquinol throughout Japan. Immediately after the stop of its sale, the continuous occurrence of SMON suddenly stopped. (Fig. $3)^{2)}$ This was regarded as the final proof of clioquinol intoxication. In 1972, the SMON Research Commission of the Japanese Government, of which I was also a member, finally announced that the SMON was nothing but the clioquinol intoxication. ${ }^{2), 3)}$

1.5. History of clioquinol. ${ }^{2), 3), 10)}$ Cloiquinol was synthesized in Switzerland in 1899, used for treatment of amoeba dysentery, and later it became to be widely used for the ordinary diarrheal patients and also to prevent traveler's diarrhea throughout the world. In Japan, it was used for the amoebadysentery in the Japanese army before and during the 2nd world war. It came back again to be widely used as a safe drug in 1960s, as a partial replacement of antibiotics, which was widely abused at that time. In this situation, clioquinol was imported or produced again in Japan as a generic drug. Unfortunately, the high dosage and long administration were rather common in Japan as mentioned above.

1.6. Aftermath of clioquinol intoxication theory of SMON. After the ban of clioquinol, SMON entirely disappeared, so no occurrence of 


\section{cases $\quad$ Fig. 3 - New SMON-patients / by month}

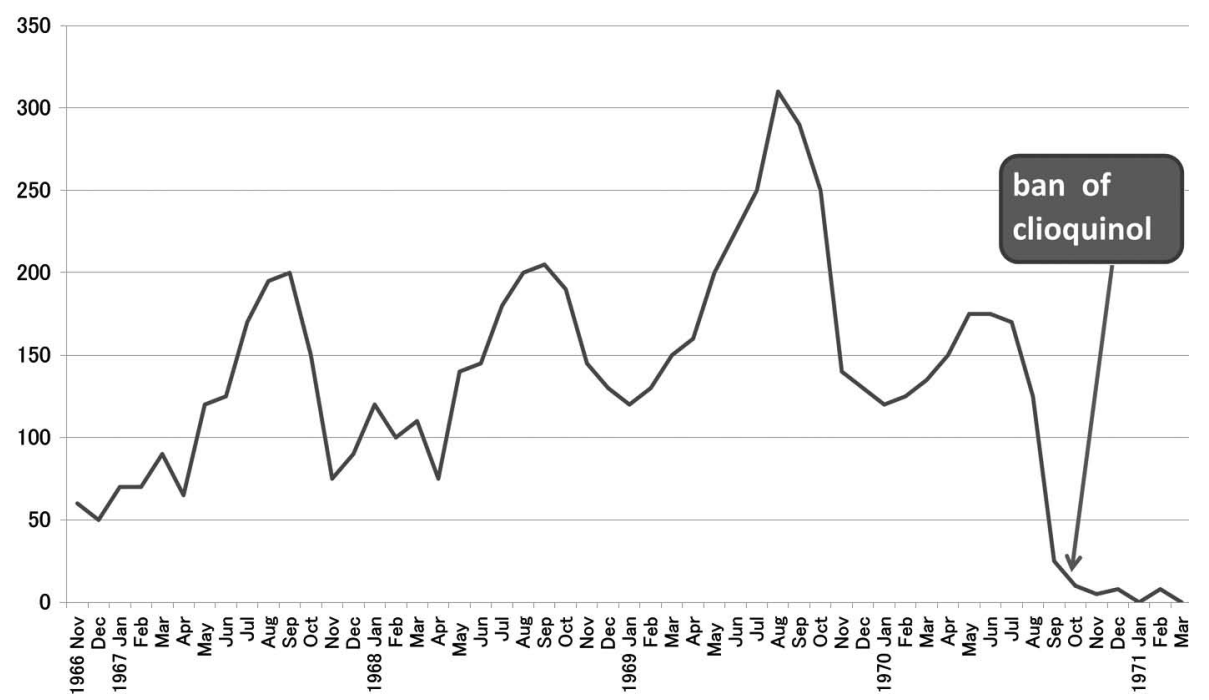

Fig. 3. New SMON-patients/by month. Continuous outbreak of SMON throughout Japan stopped in Sep. 1970 by ban of clioquinol-sale. $^{2)}$

SMON was reported thereafter until now. (Fig. 3) Due to the result of this large-scale "tragic experiment", the clioquinol intoxication theory became widely accepted throughout the world.

In 1972, roughly 7,000 patients with SMON accused the pharmaceutical companies in trials asking for the compensation of their health damage in several courts in Japan. After several years, all trials were settled by reconciliation in the courts, accepting the clioquinol intoxication theory.

In addition, the new policy to relieve many suffering patients from many intractable diseases was started by the Japanese Government. On the petition of the patients-union of SMON, the government issued ample funds to organize the nation-wide research group in 1969, which achieved the brilliant conclusion within 3 years. This success has stimulated the general sentiment, that any intractable diseases can be settled, if ample research funds are issued for organizing the nation-wide research group. This countermeasure was inaugurated in 1972, through the decision of the Prime Minister Kakuei Tanaka, so that the new policy for the several intractable diseases started, along with the support for the expenses of the treatment. These countermeasures are still operating at present, and the many splendid results were achieved. Indeed, many intractable diseases were settled and many new effective drugs were introduced through the achievements of the nation-wide research groups. This policy is internationally unique and now highly evaluated in other countries of the world. We are proud of this political decision, which greatly contributed to the progress that occurred in medical research fields, especially in neurology.

1.7. Conclusion of SMON affair. ${ }^{2)}$ The history of SMON affair was presented here from the beginning to the end, in which I was at the center. The history of nation-wide researches on SMON was an incomparable teacher instructing that any effective drugs could give harmful effect on human body. The settlement of SMON affair was a big event in Japanese medical practice, in terms of the fact that Japanese practitioners and researchers themselves found the disease and gave answer in a rather short period. The success should be regarded as a milestone of the improvement of the scientific level of Japanese medicine.

\section{Resurgence of beriberi $(1973-75)^{11-13)}$}

2.1. Introduction. Beriberi is the disease caused by the thiamine (vitamin $\mathrm{B}_{1}$ ) deficiency. Since Edo era (1603-1868), beriberi became gradually prevalent in Japan, especially among rich people. At least two Shoguns (the Governors of Japan in the Tokugawa period) died of beriberi. In the Meiji and 


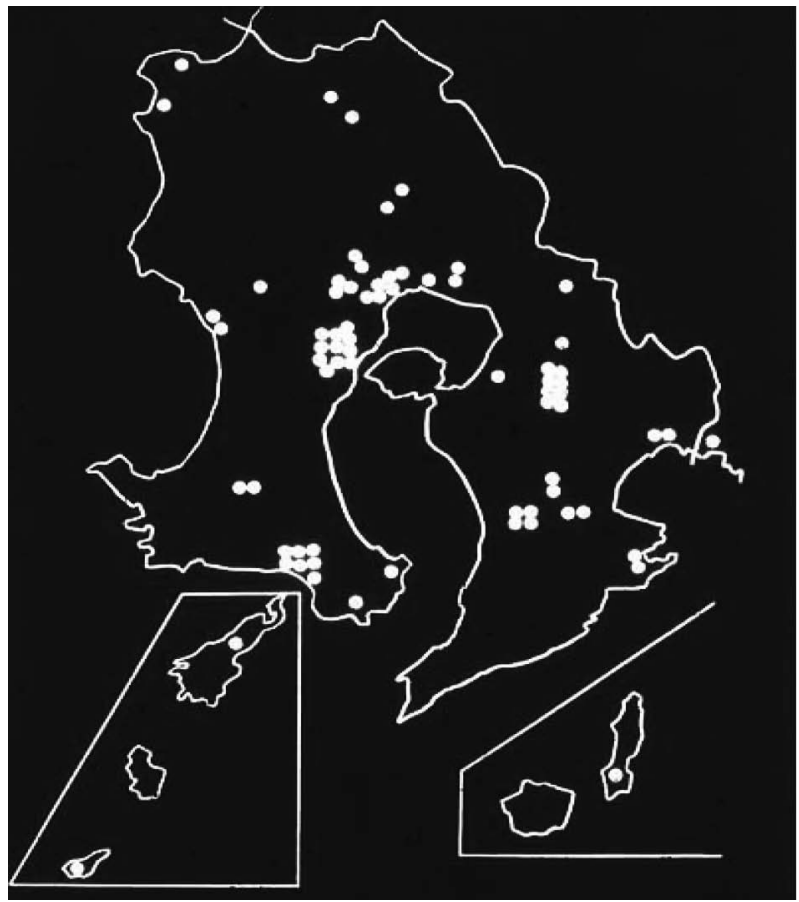

Fig. 4. Beriberi-resurgence in Kagoshima 1973-75. Many beriberi patients were distributed throughout Kagoshima. ${ }^{11)}$
Taisho era (1868-1926) beriberi was a nation-ruining disease, along with tuberculosis and syphilis. In accordance with the progress of medicine and through the nation-wide endeavor against these diseases, beriberi disappeared entirely by the end of the 2nd world war, so that doctors in 1950s knew beriberi only through the textbook and had rarely encountered with beriberi patients in daily practice. It was therefore a surprising event, to experience the unexpected resurgence in 1973-75 in Kagoshima.

2.2. Outbreak of beriberi ${ }^{11)-13)}$ (Fig. 4). In 1973-75, we experienced many young patients with oedema in both legs. At first, the etiology of oedema could not be identified and it looked to be a new disease.

The clinical features were as follows. (Fig. 5)

1) Oedema in the legs, sometimes also on the face.

2) Sensory disturbance of the distal extremities with diminished tendon reflexes, sometimes associated with peri-oral dysesthesia.

3) Grasp-pain on both calves.

4) Low diastolic blood pressure and tachycardia with heart dilatation. (Fig. 6)

5) No signs of inflammation and no leukocytosis. Later the cardiological examination revealed the high cardiac output in these patients. Theoretically this high cardiac output was compatible with beriberi, hyper-thyroidism and some other special conditions. Since other conditions except for beriberi

\section{Cases}

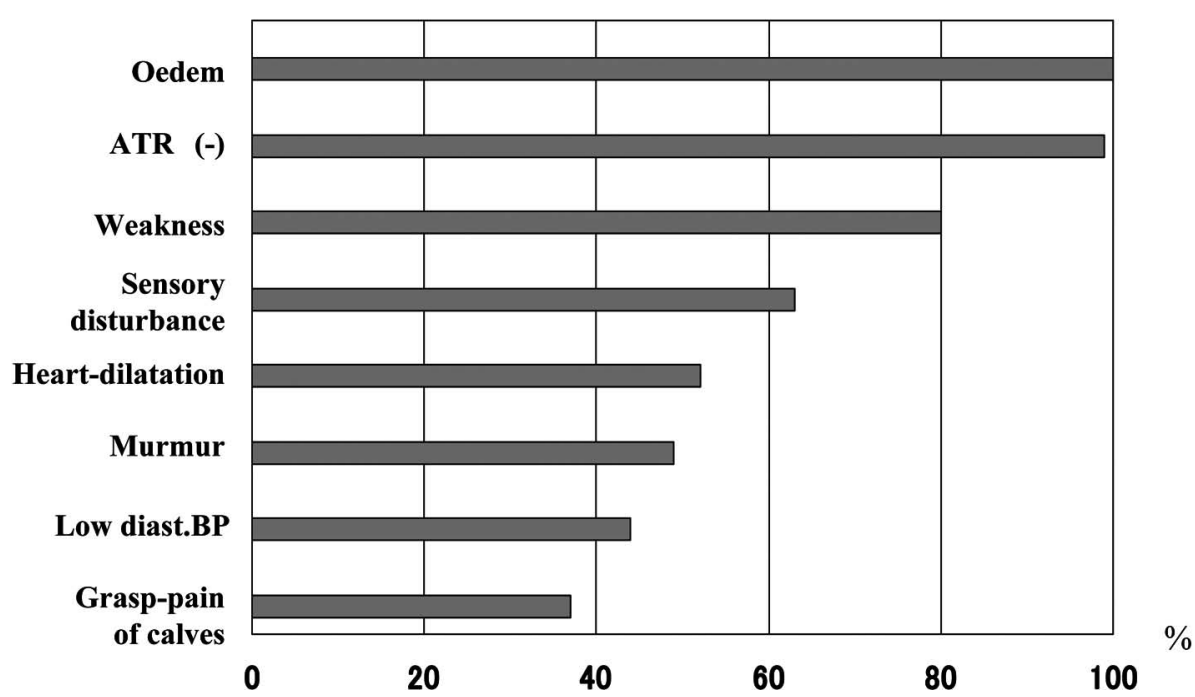

Fig. 5. Symptoms of beriberi. The incidence of each symptoms among the patients found in Kagoshima 1973-75. ${ }^{11)}$ 

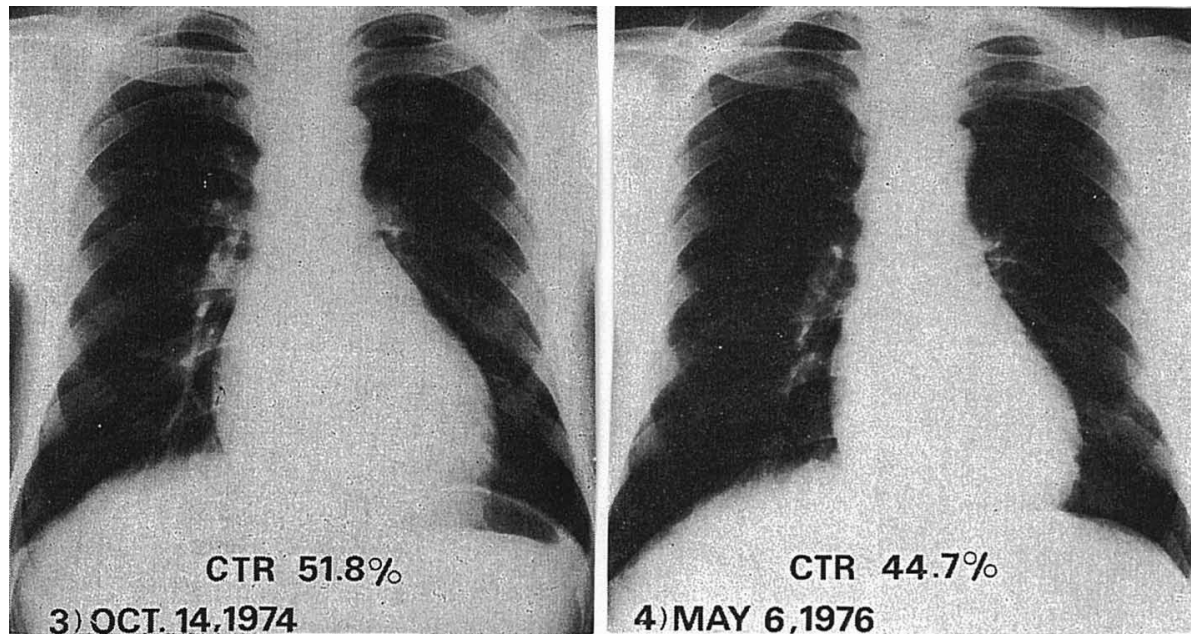

Fig. 6. Chest X ray of a patient with beriberi, before and after the thiamine treatment. Heart dilatation was recovered after the thiamine treatment. ${ }^{13)}$

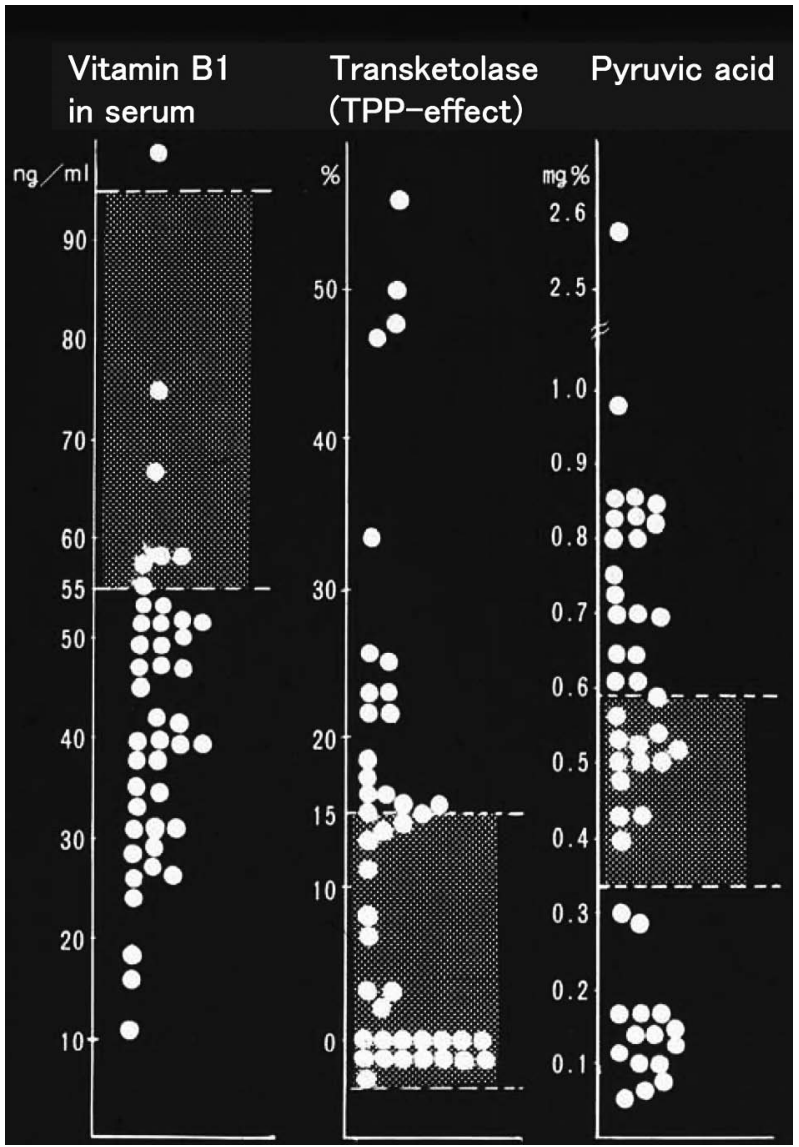

Fig. 7. The laboratory findings in serum of the beriberipatients. ${ }^{11)}$ Vitamin $\mathrm{B}_{1}$ level was lower in $87 \%$, TPP-effect on transketorase was higher in $39 \%$ and pyruvic acid level was higher than normal in $43 \%$ of patients with beriberi. were excluded by clinical and laboratory findings, we checked whether it was beriberi or not.

Based chiefly on the clinical findings, I became confident that this disease could be the long-forgotten beriberi. In addition, all clinical, biochemical and nutritional data were compatible with those of beriberi, i.e. the level of thiamine in serum was low, TPP (Thiamine pyrophosphate) effect on transketolase was marked, and pyruvic acid level in the blood was high (Fig. 7). ${ }^{11), 12)}$ By nutritional studies, it was found that the intake of thiamine was low as compared with that of carbohydrate in the patients. After the definite diagnosis was made, I undertook the epidemiological study in Kagoshima, in order to clarify the spread of the disease.

According to our study, more than 130 cases of beriberi were found in 1973-75, more in males than in females, and mainly among young people, especially high-school boys and girls.

All symptoms (Fig. 4) disappeared promptly by the administration of thiamine.

2.3. Background of the resurgence of beriberi. Until the end of the 2nd world war, Japanese people had a traditional custom to eat mainly wellpolished rice, of which the thiamine-rich mantle had been scraped off. This custom was the cause of florid beriberi-outbreak at that time. After the end of the last world war, due to the shortage of all foods, many people suffered from malnutrition which overwhelmed the manifestation of beriberi. 
Although beriberi had been forgotten, still the thiamine intake was low compared with carbohydrate even in recent years without any manifest symptoms of beriberi.

In addition, artificial sweeteners were forbidden in 1972 due to the danger of inducing cancer, so that real carbohydrate in soft drinks became increased.

The instant noodles, in which the thiamine content was clearly low relative to carbohydrate content, became also widely consumed at that time. For example, some young men living alone, took "instant noodle" at every meal, and then finally suffered from beriberi in a few months.

2.4. Aftermath of the revival of beriberi in Japan. Faced with this situation, I treated the patients with thiamine with a brilliant outcome. Also to prevent the outbreak of beriberi entirely, I undertook a big scale-campaign to distribute information about beriberi for people of Kagoshima to be aware of its resurgence.

After we recommended the city-authorities to revise the arrangements of soft-drink vending machines, so that many sugar-containing soft drinks were replaced by fruits juice, milk and tea, the resurgence of beriberi gradually disappeared.

Since this outbreak of beriberi was reported not only in Kagoshima but also in Kumamoto, Tottori, Tokyo and in many other areas in Japan, many Japanese became aware of this revival. We should never forget beriberi as far as we eat well-polished rice as the main food.

2.5. Studies in Asian countries. ${ }^{14)}$ To compare this resurgence of beriberi in Japan with other countries, I undertook epidemiological studies of prevalence of beriberi in several rice eating Asian countries and districts (Philippines, Indonesia, Thailand, and Taiwan) where rice is consumed as the main food. As a result, I found that there were no pure form of beriberi but found a large number of malnourished patients. Indeed, the thiamine contents of the rice in the market of the targeted countries and districts were higher on the average than those in Japan. Therefore, I became confident that beriberi is rather specific for Japanese, who mainly eat well-polished rice as the main food. ${ }^{14)}$

2.6. History of studies on beriberi in Japan. Until the Meiji era (1868-1912), the cause of beriberi remained obscure, though its prevalence was very high. Around that period, the development of the modern medicine started in Japan under the guidance of German professors who came to Japan. They tended to assume the importance of then flourishing bacteriology, so that they thought that beriberi in Japan might be due to a specific bacillus. Thereafter many leaders of Japan, especially in the Army and the national medical schools believed the suggestion of these German professors so that they imagined that beriberi bacillus may exist. In fact, there were many reports in Japan suggesting the beriberi-bacillus.

On the contrary, Dr. Kanehiro Takaki, who was the head of the medical department of Japanese Navy and later a founder of Jikei Medical School, insisted that beriberi must be induced by the diet related factor, based on the large scale experiment achieved in the Navy in 1884 .

In 1883, a warship "Ryujo" made a voyage to Sidney, Chili and Hawaii, in which the crew was fed with a traditional Japanese diet, and more than half of the crew suffered from beriberi. Knowing this fact, he planned the same voyage by a warship "Tsukuba" with a European style diet. As he expected, only a few beriberi patients were reported. Therefore he reached to the conclusion that beriberi was caused by the traditional Japanese diet, and he revised the diet in the navy to the European style, then, beriberi disappeared from the Japanese navy.

In contrast, in the army, the medical leaders including Dr. Rintaro Mori, the head of the medical department of the Japanese army, continued to believe that the beriberi should be infectious in origin, and refused to accept Takaki's food theory as an old bigotry. Japanese soldiers in the army were fed with a typical Japanese diet consisting mainly of polished rice which was regarded as a gorgeous treat.

Although more than 10 years had passed after the food theory was proposed by Dr. Kanehiro Takaki, many soldiers in the army died of beriberi the number of whom was comparable with that of direct war-deaths during the Russo-Japanese war (1904-05), whereas no beriberi death occurred in the navy.

In 1896, Dr. Christian Eijckman had succeeded in producing beriberi in chickens by feeding with polished rice and confirmed the curing effect of rice bran, the effective constituent of which was later clarified as a kind of vitamin (thiamine) by Casimir Funk in 1912.

It should be stressed that Dr. Umetaro Suzuki was the one of the discoverers of vitamin $\mathrm{B}_{1}$. After the role of the vitamin in beriberi was internationally 


\section{case-No. Peripheral
blood}

CSF
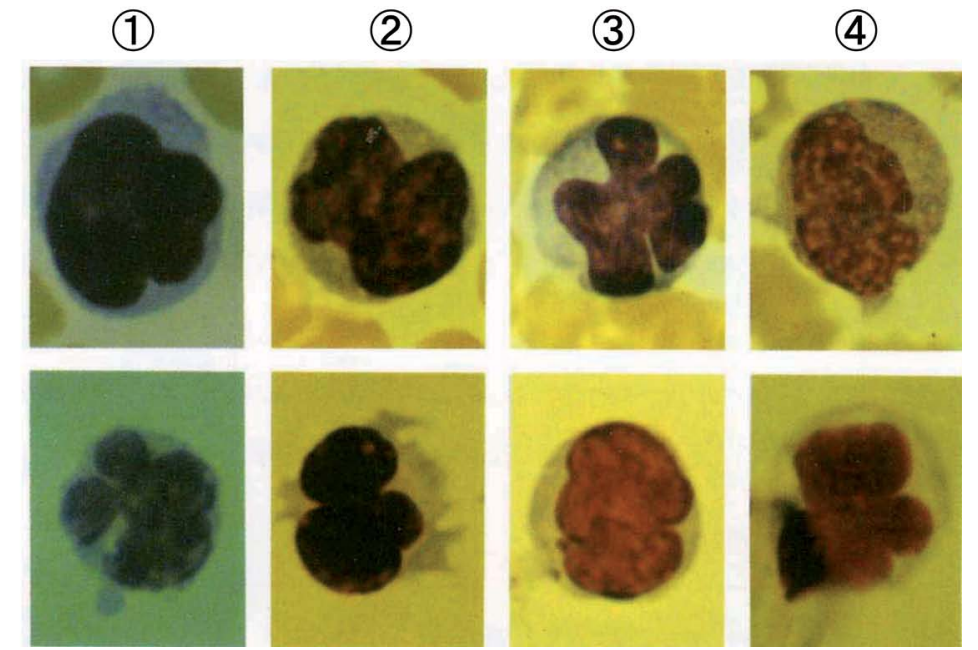

Fig. 8. ATLL-like cells in the peripheral blood and the CSF of HAM-patients. The abnormal cells were identical with those of ATLL. ${ }^{22)}$

accepted, all Japanese leaders became convinced that beriberi was caused by thiamine deficiency. Thereafter the Japanese government had tried to suppress the outbreak of beriberi, establishing the system to educate the dieticians, so that the occurrence of beriberi gradually diminished and finally disappeared. We should never forget the brilliant results of epidemiological experiment supervised by Dr. Kanehiro Takaki, in order to avoid the resurgence of beriberi in the future.

\subsection{Conclusion of the beriberi affair.}

I experienced the resurgence of beriberi in the early 1970s in Japan. As far as we eat well-polished rice as a main food, we should always be aware of beriberi.

\section{A new spinal cord disease, HAM (HTLV-I associated myelopathy) ${ }^{15)-17)}$}

3.1. Introduction. The history of HTLV-I associated myelopathy (HAM) dates back to 40 years ago. When I accepted the professorship of the Department of Internal Medicine, Kagoshima University, I promptly started epidemiological studies of all neurological diseases in Kagoshima prefecture. In the accumulated data, a group of sporadic spastic paraparesis was noted. ${ }^{15)}$

Our research was started to elucidate the cause of this particular disease, but the cause remained unknown for many years.

3.2. The symptoms of HAM. 15),17) The characteristic features of this disease were as follows:

1) Gradual onset followed by slow progression.
2) Sporadic spastic paraparesis.

3) Adult onset without any hereditary transmission.

4) Prominent pyramidal tract signs and mild sensory and sphincter disturbances.

5) No cerebral symptoms, no sign of common inflammation.

The prevalence rate of this new disease in Kagoshima prefecture was estimated to be $55 / 1,700,000$, whereas that of a most popular muscle degenerative diseases, Duchenne type of muscular dystrophy was 29/1,700,000.

We recognized immediately that this was a new disease.

3.3. Hints for the cause of HAM. Since the 1970s a peculiar type of lymphoma had been known to be prevalent in southern Kyushu including Kagoshima. This lymphoma was characterized by proliferation of malignant cells in peripheral blood with the coexistence of lymphomas of various size. This was named as "cauliflower like-leukemia", after the appearance of the nucleus of the proliferated lymphocytes as reported by the Cancer Research Institute of Kagoshima University. ${ }^{18)}$ Independent from this report, Dr. Kiyoshi Takatsuki, Kyoto University, found some patients suffering from similar lymphoma in Kyoto, who were born in Kagoshima prefecture. He noticed that this was of a $\mathrm{T}$ cell origin, identical with those reported in Kagoshima, and he gave the name "Adult T cell lymphoma/leukemia (ATL)".19) Following this discovery, Prof. Yorio Hinuma, a virologist in Kyoto University and Dr. Mitsuaki Yoshida, 


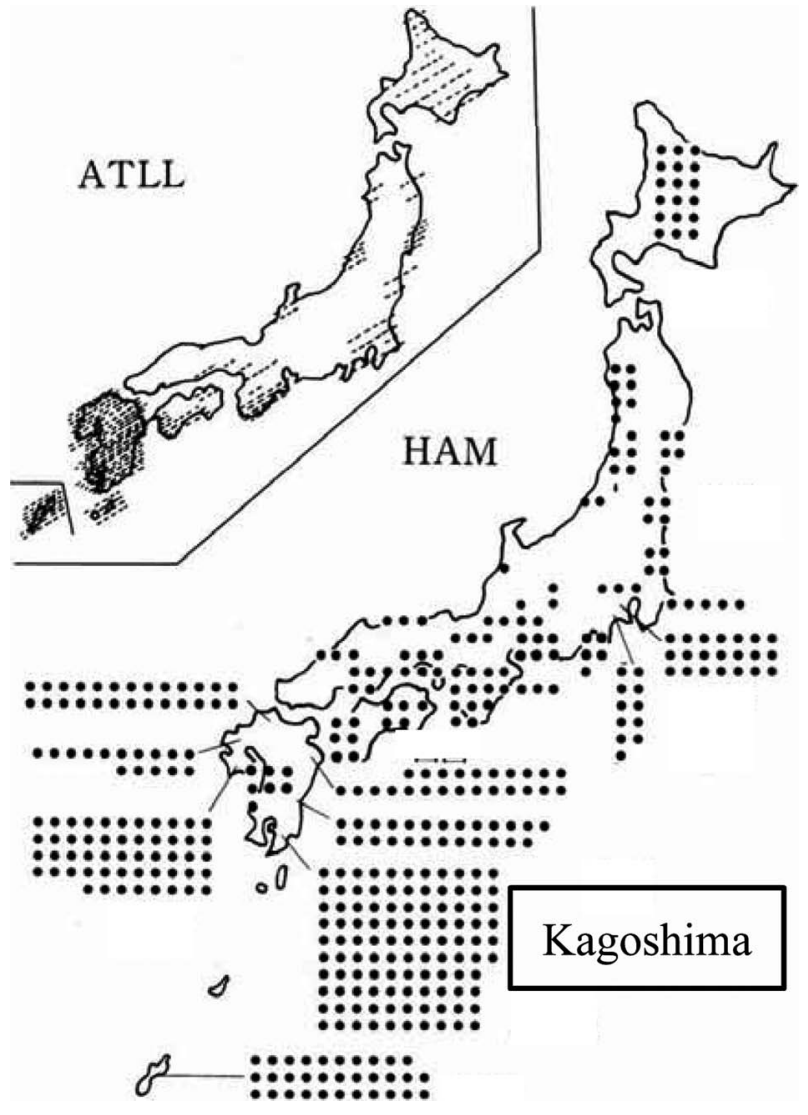

Fig. 9. Prevalence of HAM and ATLL throughout Japan. ${ }^{22)}$ The prevalence of both diseases was similar and dominant in south-western areas of Japan.

found its causative virus and named it as "human T lymphotropic virus type I (HTLV-I)". 20$), 21$ )

By chance in 1984, we noticed the presence of "adult T cell leukemia-like cells" in blood and CSF of patients with the above mentioned neurological disease, without any signs of malignant proliferation and lymphoma. (Fig. 8) In addition, we found that the titer of the antibody against the HTLV-1 virus in the blood and the CSF was definitely increased. (Fig. 7)

"Our disease" seemed, however, to be different from ATL not only hematologically but also clinically. This fact enabled us to conclude that our disease and ATL are different from each other but both occur among the carriers of HTLV-I. ${ }^{17)}$

Neuropathological investigation of "our disease" confirmed the systemic degeneration of bilateral long tracts, predominantly of the pyramidal tract of the spinal cord. In addition the scattered perivascular lymphocyte-infiltration was also found as a characteristic feature in spite of the absence of the abnormal proliferation of $\mathrm{T}$ lymphocytes in the blood and the CSF. Therefore, we were confident that this neurological disorder should not be classified as ATL but should be considered as a particular type of myelopathy caused by HTLV-I.

Our first report in 1987 was based on the data obtained from 85 patients; 58 males and 19 females. The age of onset was from 6 to 75 years. ${ }^{17)}$ Through a nationwide survey, the distribution of HAM was clarified as shown in Fig. 9.

The report gave a strong impact on the medical society, because this was the first example that the same virus can induce entirely different diseases in terms of both the clinical course and the pathological features.

3.4. Relationship of HAM with tropical spastic paraparesis (TSP) (Fig. 10). Interestingly, a similar spastic paraparesis had been known to be prevalent in many tropical areas in the world, which had been known as tropical spastic paraparesis (TSP). It was found that many patients with TSP had very high titer of antibody to HTLV-I in 1985. ${ }^{22)}$

In 1992, an International symposium on HAM/ TSP was held in Kagoshima under the auspices of the WHO and the conclusion was obtained that both diseases were identical.

3.5. Geographical difference in Japan. Roughly speaking, it was estimated that one case among 2,000 asymptomatic antibody-carriers will develop HAM, and another one among 1,000 ATL. It was remarkable that the prevalence of HAM and ATL was very high in Kyushu, the southern part of Japan, compared with that in the central and northeastern parts of Japan. Since the geographical difference of HAM and ATL was so high between northern and southern Japan, we challenged the elucidation of the reason of the geographical difference. (Fig. 9)

Consequently, it was suggested that this difference originated from the race difference among Japanese, which consist of 2 components, one of old Mongolian (Johmon: which immigrated to Japan from Eurasia continent before the glacial age), and the other Neo-Mongoloid (Yayoi) which immigrated to Japan about 2,000 years ago after the glacial age. The HTLV-1 virus remained mainly in the former Johmon group by transmission through lactation from HTLV-I sero-positive mothers, who lived pre- 


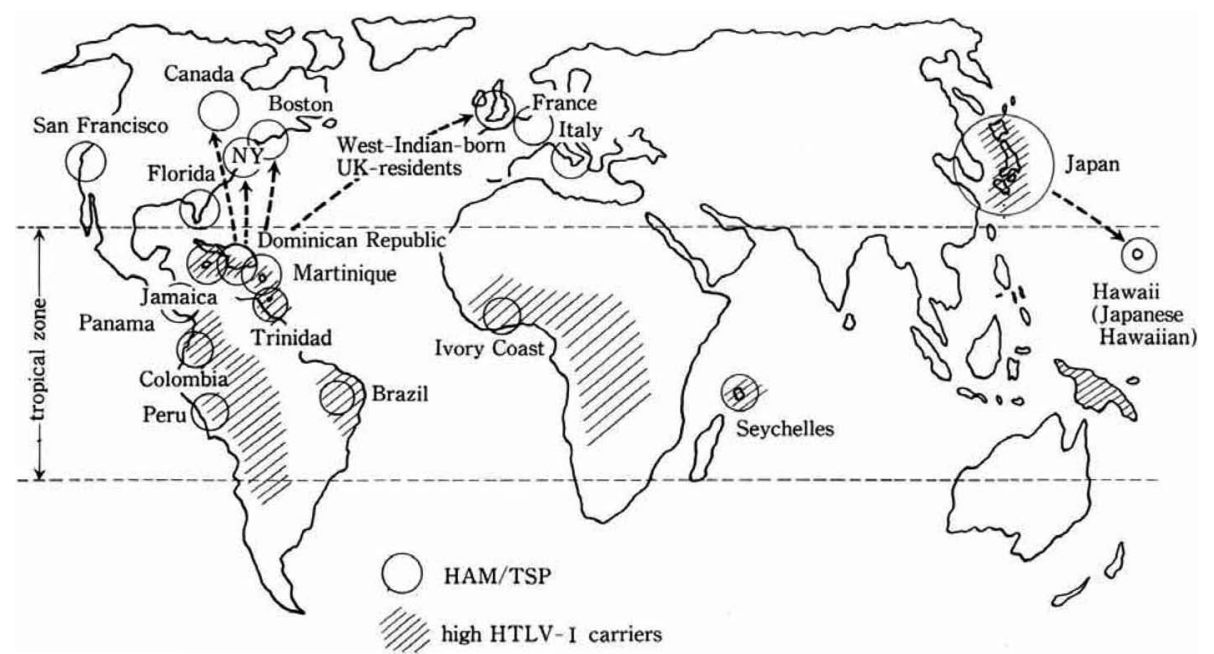

Fig. 10. Prevalence of HAM/TSP. ${ }^{23)}$

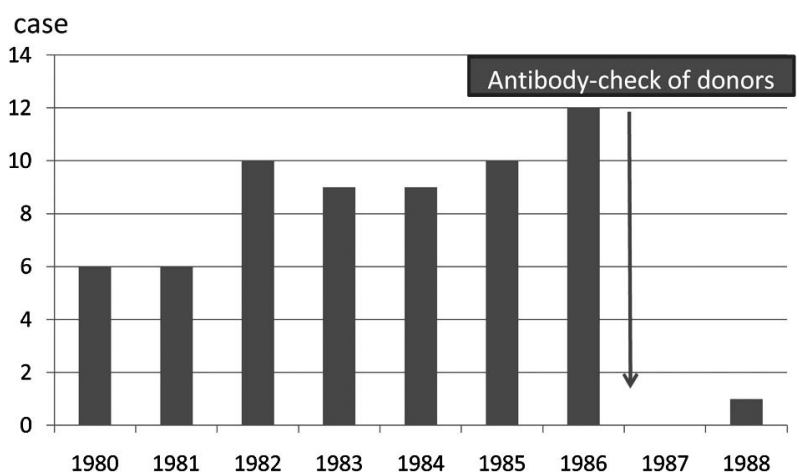

Fig. 11. HAM-patients after blood transfusion. ${ }^{24)}$

HAM patients after blood transfusion disappeared by the antibody-check of donors initiated in 1986 on our proposal.

dominantly in southern Japan, whereas the latter Yayoi group in the central and northern Japan. That difference might be the main reason for the geographic difference in the prevalence of HAM and ATL within the Japanese.

\subsection{Transmission of HTLV-I through the} blood transfusion (Fig. 11). ${ }^{23)}$ It was also suggested that a certain group of HAM patients had received blood transfusion before the onset of this disease. Based on our suggestion that the incidence of HAM may be due to the blood transfusion, the Japanese government stopped transfusion from seropositive donors, and the transmission of the disease through blood transfusion is completely prevented now.
3.7. Mother-child transmission of HAM. ${ }^{24)}$ Through our studies on HAM, the mother-child transmission was strongly suggested. Furthermore, it became clear that the virus is transmitted through mothers' milk. Now, it is strongly recommended to the HTLV-I sero-positive mothers not to give the direct lactation to suckling babies.

3.8. Relationship between HAM and multiple sclerosis. Since several reports suggested that the antibody against HTLV-1 could be found in some patients with multiple sclerosis (MS) in 1980s, HTLV-I became the biggest topic in the world, because MS is the most common neurological disease in the Western countries. Later, however, this was proved not to be true by many researches. Now the patho-mechanism of HAM has become clear, especially from neuro-immunological aspects, and the name of HAM is now well accepted internationally.

3.9. Conclusion of HAM affair. ${ }^{16), 25)}$ Looking back over the history of our clinical studies on HAM, I have a strong feeling that a locally found minor disease became an important one of the international interest through our studies. This is a good example of our slogan "to keep in mind to send message of scientific progress from the local area to the international stage". Further studies on the detailed patho-mechanism of HAM and its treatment are now in progress by the research group headed by former Prof. Mitsuhiro Osame of Kagoshima University. 


\section{References}

1) Igata, A. and Toyokura, Y. (1970) SMON in JapanZur Frage der Chinoform Vergiftung-, Muench. Med. Wschr. 113, 1062-1065.

2) SMON Research Commission (1985) History and review of SMON research. Jap. Ministry of Health and Welfare.

3) Pallis, C.A. and Dewis, P.D. (1974) Neurological complications of clioquinol therapy. The Neurology of Gastrointestinal Diseases. W.B. Saunders Co. pp. $179-185$.

4) Tsubaki, T., Toyokura, Y. and Tsukagoshi, H. (1965) Subacute myelo-optico-neuropathy following abdominal symptoms - a clinical and pathological study. Jap. J. Med. 4, 181-184.

5) Inoue, K. (1970) A virus unique to SMON patients. Annual Report of the Institute of Viral Research, Kyoto Univ. 13, 71-72.

6) Igata, A., Hasebe, T. and Tuji, T. (1970) Two SMON-patients excreting green urine. Nihon Iji Shinpo 2412, 25-28 (in Japanese).

7) Tamura, Z., Yoshioka, S., Imanari., T., Furuya, J., Kusaka, J. and Sejima, K. (1973) Identification of green pigment and analysis of clioquinol in specimens from patients with subacute myelo-opticoneuropathy. Clinica Chemica Acta 47, 13-20.

8) Tsubaki, T. (1970) Proposal to the Ministry of Health \& Welfare to inhibit clioquinol-sale in Japan. 6, Aug. 1970 (in Japanese).

9) Igata, A. and Tsubaki, T. (1972) Comparative study on the pathogenesis of SMON, Final report, WHO Exchange of Research Workers Grant.

10) Igata, A. (1972) Clinical aspects of SMON - Progress of neurological researches, Shinkei Kenkyu No Shinpo 16, 853-856 (in Japanese).

11) Arima, H. (1980) The clinical studies on the polyneuropathy with oedem. Kagoshima Igaku Zassi 31, 689-720 (in Japanese).

12) Kuriyama, M., Yokomine, R., Arima, H., Hamada, R. and Igata, A. (1980) Blood vitamin $\mathrm{B}_{1}$ transketorase and thiamin pyrophosphate effect in beriberi patients with studies, employing discriminant analysis. Clinica Chimica Acta 108, 159-168.

13) Nishi, S., Arima, H., Shinyashiki, S., Uchimura, H., Katanazako, H., Tanaka, H. et al. (1977) Clinical studies on beriberi heart. J. Molecular and Cellular Cardiology 9 (Suppl.), 59-61.

14) Igata, A. (1981) Comparative studies on vitamin-B deficiency in southern Asian countries, presented in the 22nd Congress of Japanese Society of Neurology.
15) Osame, M., Arima, H., Norimatu, K., Kawahira, M., Ohkatu, Y., Nagamatu, K. et al. (1975) Epidemiological studies on muscle atrophy in southern Kyushu, Jap. J. Med. 14, 230-231.

16) Igata, A. (1987) New spinal cord disease. Kagaku 57, 811-816 (in Japanese).

17) Osame, M., Matsumoto, M., Usuku, K., Izumo, S., Amitani, H., Ijichi, N. et al. (1987) Chronic progressive Myelopathy associated with elevated antibody to human $\mathrm{T}$ lymphotropic virus type-I and adult T cell leukemia-like cells. Annal. Neurol. 21, $117-122$

18) Nomura, K. and Matsumoto, M. (1981) Clinical features of adult $\mathrm{T}$ cell leukemia in Kagoshima, the southernmost district in Japan - Comparison with T-cell lymphoma. Act. Hematol. Jap. 44, 1444 1457.

19) Uchiyama, T., Yodoi, J., Segawa, K., Takatsuki, K. and Uchino, H. (1977) Adult T cell leukemia. Blood 50, 481-482.

20) Hinuma, Y., Nagata, K., Hanaoka, M., Shirakawa, S. and Miyoshi, I. (1981) Adult T cell leukemia-Antigen in an ATL cell line and detection of antibodies to the antigen in human serum. Proc. Natl. Acad. Sci. USA 78, 6476-6480.

21) Yoshida, M., Miyoshi, I. and Hinuma, Y. (1982) Isolation and characterization of retrovirus from cell lines of human adult $\mathrm{T}$ cell leukemia and its implication in the disease. Proc. Natl. Acad. Sci. USA 79, 2031-2035.

22) Gassain, A., Vernant, J.C., Maurs, L., Barim, F., Gouct, O., Calender, A. et al. (1985) Antibodies to Human T-lymphotropic virus type I in patients with Tropical Spasticc Papaparesis. Lancet 2, 407-413.

23) Osame, M., Izumo, S., Igata, A., Matsumoto, M. Sonoda, S., Tara, M. et al. (1986) Blood transfusion and HAM. Lancet 2, 104-105.

24) Osame, M., Igata, A., Usuku, K., Rosales, R.L. and Matsumoto, M. (1986) Mother-to-child transmission in HTLV-I associated myelopathy. Lancet 1 , 106.

25) Igata, A. (1993) HTLV-I associated Myelopathy. In Amyotrophic Lateral Sclerosis - from Charcot to the present and into the future. (ed. Rose, F. C.). Smith-Codon.

(Received Oct. 26, 2009; accepted Feb. 3, 2010) 


\section{Profile}

Akihiro IGATA was born in 1928 and started his clinical research career at the department of internal medicine of University of Tokyo and he received the degree of medical doctor in 1960 by a doctoral dissertation titled "studies on the neuro-humoral theory-distribution of cholinesterase in the peripheral autonomic nerves-".

In 1961-62 he was engaged in clinical research in university of Würzburg in Germany. In 1965 he became an assistant of the newly established department of neurology in the University of Tokyo. In 1971 he was appointed as Professor of the department of internal medicine, Kagoshima University and thereafter was engaged in the clinical researches of neurological diseases for many years.

In 1987 he was elected as the president of Kagoshima University. In 1993, he

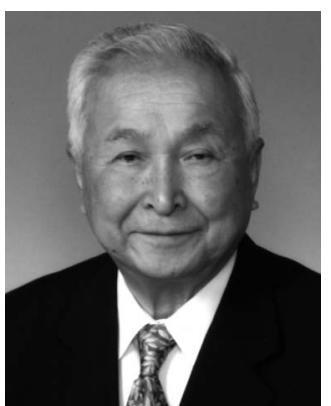
moved to Nagoya to establish the national center of geriatrics and gerontology in Nagoya.

In 1997, he was nominated as the president of Aichi Comprehensive Health Science Center in Nagoya. Since 2002 until now, he has been the president of Nagoya University of Arts \& Sciences.

In 1989 he received the H. Noguchi memorial prize and in 1992 he was given the medal with purple ribbon by the Japanese government. 